\title{
Adaptive Detector Arrays for Optical Communications Receivers
}

\author{
V. Vilnrotter ${ }^{1}$ and M. Srinivasan ${ }^{1}$
}

\begin{abstract}
The structure of an optimal adaptive array receiver for ground-based optical communications is described and its performance investigated. Kolmogorov phase screen simulations are used to model the sample functions of the focal-plane signal distribution due to turbulence and to generate realistic spatial distributions of the received optical field. This novel array detector concept reduces interference from background radiation by effectively assigning higher confidence levels at each instant of time to those detector elements that contain significant signal energy and suppressing those that do not. A simpler suboptimum structure that replaces the continuous weighting function of the optimal receiver by a hard decision on the selection of the signal detector elements also is described and evaluated. Approximations and bounds to the error probability are derived and compared with the exact calculations and receiver simulation results. It is shown that, for photoncounting receivers observing Poisson-distributed signals, performance improvements of approximately $5 \mathrm{~dB}$ can be obtained over conventional single-detector photoncounting receivers, when operating in high background environments.
\end{abstract}

\section{Introduction}

Ground-based reception of optical signals from space suffers from degradation of the optical phase front caused by atmospheric turbulence. This leads to a reduction in the effective diameter of the receiving telescope and to random fluctuations of the receiver's point-spread function (PSF) in the focal plane. For example, the diffraction-limited field of view (FOV) of the receiving telescope can be taken to be approximately $\theta_{d l} \cong \lambda / D_{R}$, which, for a 3 -m aperture and $1-\mu \mathrm{m}$ wavelength, translates to $0.33 \mu \mathrm{rad}$. If the effective focal length of the telescope is $6 \mathrm{~m}$ (implying an $\mathrm{F} / 2$ instrument), then a diffraction-limited PSF of $2-\mu \mathrm{m}$ diameter, or $0.002 \mathrm{~mm}$, will be produced in the focal plane. Thus, under ideal conditions, a very small detector could be used to collect virtually all of the signal energy while at the same time spatially filtering out most of the background radiation. However, atmospheric conditions rarely permit diffraction-limited operation of large telescopes; even under good nighttime seeing conditions, the phase of the received signal field tends to become uncorrelated over distances greater than $20 \mathrm{~cm}$, deteriorating to as little as 2 to $4 \mathrm{~cm}$ during the day [1]. Under these conditions, the dimensions of the PSF in the focal plane tend to increase inversely with coherence length, as if the dimensions of a diffraction-limited telescope were correspondingly reduced; the telescope still collects all of the signal energy propagating

\footnotetext{
${ }^{1}$ Communications Systems and Research Section.

The research described in this publication was carried out by the Jet Propulsion Laboratory, California Institute of Technology, under a contract with the National Aeronautics and Space Administration.
} 
through its physical aperture, but the collected signal energy is redistributed into a much larger spot in the focal plane. Thus, the receiver's FOV must be increased proportionally to collect the signal, leading to a corresponding increase in the amount of interfering background radiation.

An example of the increase in the effective dimensions of the receiver's point-spread function over its diffraction-limited value is shown in Figs. 1(a) and 1(b), corresponding to a 1-m aperture, 0.3-m central obstruction, and 4 -cm atmospheric coherence. Although a telescope of $1-\mathrm{m}$ diameter with a secondary obstruction of $0.3 \mathrm{~m}$ has been assumed, similar results hold for larger telescope diameters as well (such as 3-m- or 10-m-diameter telescopes) as long as the focal-plane signal distribution is dominated by turbulence effects. Since the diffraction-limited PSF is inversely proportional to the telescope diameter, this condition generally will be satisfied for larger telescope diameters; however, the background and signal energies must be properly scaled to account for the larger collecting area. If the receiver optics are not diffraction limited but have significant imperfections, generating a PSF with dimensions that are comparable to the turbulence-induced distribution, then more detailed modeling of the signal distribution becomes necessary; that problem has not been addressed here.

In order to collect all of the signal energy, the dimensions of a single optical detector must be made large enough to encompass the degraded point-spread function as well as its random excursions in the focal plane, which tend to change on time scales of 10 to $100 \mathrm{~ms}$. Thus, the active area of the detector must be made large enough to encompass most of the signal energy most of the time. However, a large detector implies a large receiver field of view, which in turn implies a corresponding increase in the amount of background radiation admitted into the receiver. That, in turn, degrades communications performance. These problems are effectively mitigated by the use of a high-speed photon-counting detector array together with high-speed digital electronics capable of performing the signal-processing functions required for optimum or near-optimum receiver performance.

A conceptual block diagram of an optical photon-counting array receiver is shown in Fig. 2. The receiver consists of a collecting aperture and optics to focus the collected fields onto the focal plane, where a detector array capable of counting individual photons responds to the impinging fields. The individual output voltages from every element of the array are converted to numerical samples, which then are observed by a high-speed digital signal-processing assembly that performs the required mathematical operations, called algorithms, in order to optimize detection performance. The derivation and evaluation of the optimum detection algorithms, and of simpler but effective suboptimum structures, is the subject of this article.

\section{Optical Direct Detection With Focal-Plane Arrays}

In the following analyses, we shall assume that the optical bandwidth of the receiver is great compared with its electrical bandwidth, so that a multimode assumption can be applied to both the signal and background fields. It has been shown that multimode Gaussian fields with suitably small average modal noise count generate approximately Poisson-distributed random-point processes at the output of an ideal photon-counting detector [2]. This model is reasonable for communications systems operating even at megabit-per-second rates and justifies the use of the relatively simple Poisson model, which, in turn, often leads to mathematically tractable solutions.

\section{A. Single-Detector Model}

Suppose that a single-detector element measures the number of photons contained in the received field by producing a stream of free electrons at its output terminal in response to the absorbed photons. If the occurrence time of each pulse can be measured, and if the amplitude of each pulse is normalized to unity, then we can define a count record consisting of positive integer-valued jumps occurring each time a photon is detected. Therefore, the count record, or count accumulator function, is a monotonically increasing function that contains all of the information present in the detection process. As in [3], we can 
(a)

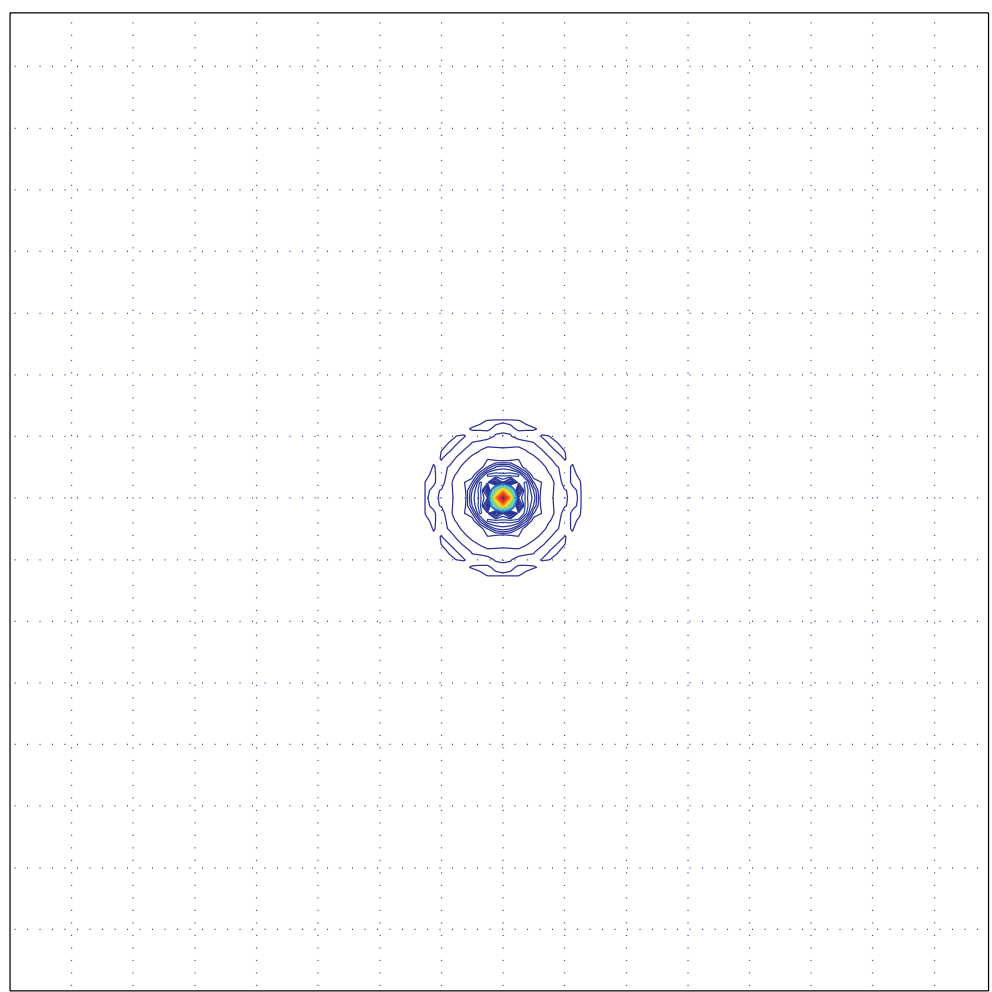

(b)

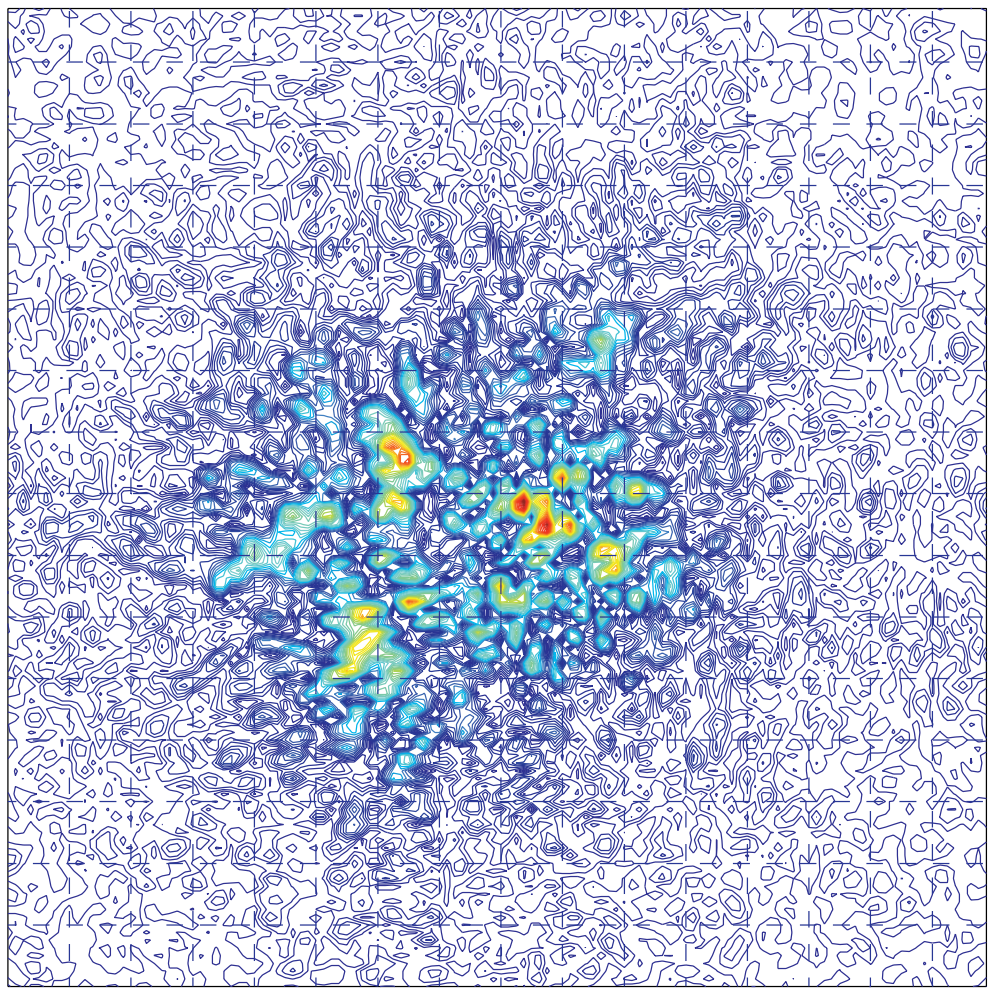

Fig. 1. Instantaneous focal-plane signal distribution (covering a $16 \times 16$ detector array): (a) for an undistorted received field corresponding to a $45 \times 45 \mu \mathrm{rad}$ FOV and (b) due to atmospheric turbulence, a 4-cm coherence length, and a $45 \times 45$ urad FOV. 


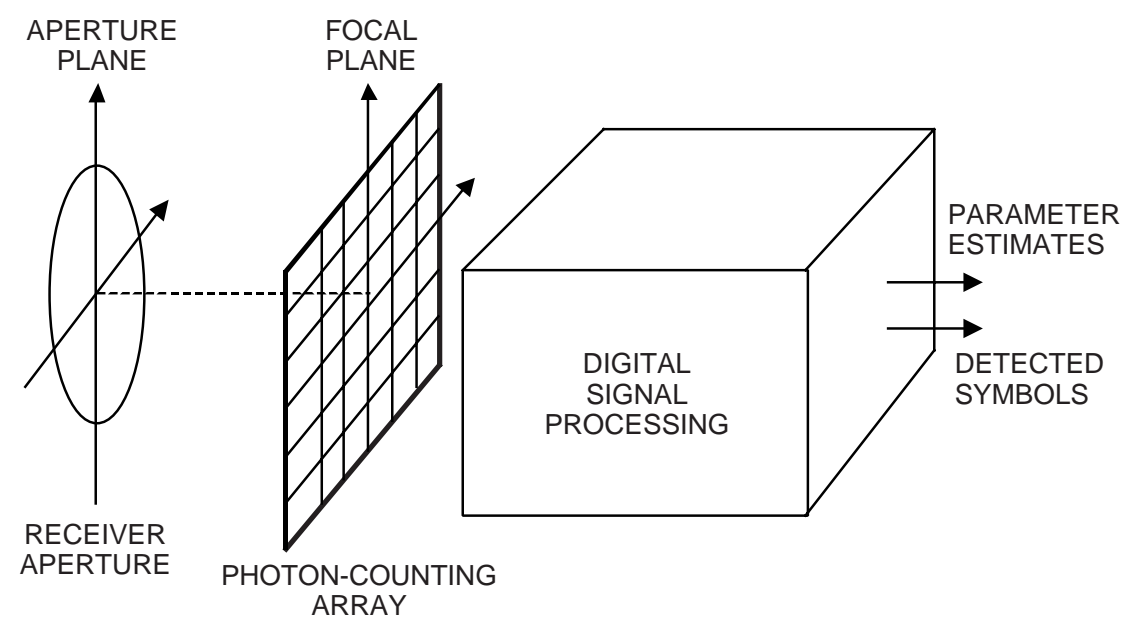

Fig. 2. Conceptual block diagram of the optical photon-counting array receiver.

associate a conditional sample function density with the detected process, conditioned on the intensity function $\lambda(t)$, defined as

$$
\begin{aligned}
p[N(t) \mid \lambda(t) ; 0 \leq t<T] & = \begin{cases}\operatorname{Pr}(N(T)=0 \mid \lambda(t)) ; & N(T)=0 \\
p_{w}\left(w_{1}, w_{2}, \cdots, w_{N}, N(T)=N \mid \lambda(t)\right) ; & N(T) \geq 1\end{cases} \\
& = \begin{cases}\exp \left(-\int_{0}^{T} \lambda(t) d t\right) ; & N(T)=0 \\
{\left[\prod_{i=0}^{N} \lambda\left(w_{i}\right)\right] \exp \left(-\int_{0}^{T} \lambda(t) d t\right) ;} & N(T) \geq 1\end{cases}
\end{aligned}
$$

where the set $\left\{w_{i}\right\}$ is the occurrence times of the detected pulses; $N(t)$ is called the count accumulator function of the process over the time interval $[0, T)$; and $\lambda(t)=\iint_{A} d x d y \lambda(x, y ; t)$, where $A$ is the detector area and $\lambda(x, y ; t)$ is the intensity process over space and time.

Given a particular realization of the intensity function, $\lambda(t)$, the joint density of occurrence times conditioned on $N$ and $\lambda(t)$ is given by

$$
p(\mathbf{w} \mid N, \lambda(t))=\frac{p(\mathbf{w}, N \mid \lambda(t))}{p(N \mid \lambda(t))}=(N !) \prod_{i=1}^{N} \lambda\left(w_{i}\right)\left(\int_{0}^{T} \lambda(t) d t\right)^{-1}
$$

where $\mathbf{w}=\left(w_{1}, w_{2}, \cdots, w_{N}\right)$. The conditional density of the total number of counts, $N$, given $\lambda(t)$, can be expressed as

$$
p(N \mid \lambda(t))=(N !)^{-1} \exp \left(-\int_{0}^{T} \lambda(t) d t+\sum_{i=1}^{N} \ln \lambda\left(w_{i}\right)\right)
$$

The expressions derived so far describe the output of a single photon-counting detector responding to optical intensity. If instead of just a single detector an array of detectors were used to detect the optical fields, then the notation must be suitably generalized to enable unambiguous description of the output of each detector element. 


\section{B. Array-Detector Model}

Consider a rectangular array of detectors consisting of $K \times L$ detector elements. For some applications, such as finding the center of the signal intensity distribution, it is important to know the location of each detector element within the array; therefore, we include the subscripts $m n, 1 \leq m \leq K$, and $1 \leq n \leq L$ to denote the position of the detector element within the array. Thus, the sample function density defined in Eq. (1) can be written as $p\left[N_{m n}(t) \mid \lambda_{m n}(t) ; 0 \leq t<T\right]$, which now represents the output of a particular element of the array. Note that $\lambda_{m n}(t)$ can be viewed as that portion of a spatially distributed intensity function intercepted by the mnth detector element,

$$
\lambda_{m n}(t)=\iint_{A_{m n}} d x d y \lambda(x, y ; t)
$$

where $A_{m n}$ is its effective area. Note that if the spatial intensity distribution is known, and the location and size of each detector element also are known, then conditioning on the spatial intensity distribution is equivalent to conditioning on the array of intensity components, each of which is still a function of time. Assuming that each array element observes the sum of a signal field plus multimode Gaussian noise field with average noise count per mode much less than one, the array outputs can be modeled as conditionally independent Poisson processes, conditioned on the average signal intensity over each detector element $[2,4]$. Hence, we denote the joint conditional sample function density of the array as

$$
p[\mathbf{N}(t) \mid \boldsymbol{\lambda}(t) ; 0 \leq t \leq T]=\prod_{m=1}^{K} \prod_{n=1}^{L} p\left[N_{m n}(t) \mid \lambda_{m n}(t) ; 0 \leq t<T\right]
$$

where $\mathbf{N}(t) \equiv\left(N_{11}(t), N_{12}(t), \cdots, N_{K L}(t)\right)$ and each component on the right-hand side of Eq. (5) is of the form defined in Eq. (1). This can be used as a starting point for problems involving hypothesis testing and parameter estimation, where the desired information is contained in the intensity distribution but only the array of count accumulator functions can be observed.

\section{Hypothesis Testing for Poisson Processes}

Consider $M$ ary pulse-position modulation (PPM) in which one of $M$ intensity functions is received and the receiver attempts to determine the correct symbol based on observations of the array of count accumulator functions over each of $M$ time slots. It is assumed that the symbol boundaries are known and that the arrival time of each detected photon and total number of detected photons can be stored for a limited duration of time necessary for processing.

With $M$ ary PPM modulation, a signal pulse of duration $\tau$ s is transmitted in one of $M$ consecutive time slots, resulting in a PPM symbol of duration $T=\tau M \mathrm{~s}$. Under the hypothesis that the signal pulse is contained in the $i$ th time slot, the signal intensity is given by

$$
\lambda^{(i)}(t)= \begin{cases}\lambda_{s}(t) & (i-1) \tau \leq t<i \tau \\ 0 & \text { else }\end{cases}
$$

After propagating through the atmosphere and through the receiving optics, this temporal intensity function is transformed into a space-time intensity function in the detector plane. The receiver also collects background energy from all directions, which is assumed to contribute an additional constant intensity of $\lambda_{b}$ per detector element. The integrated intensity over the $m n$th detector element is given by Eq. (4), which we now designate as $\lambda_{m n}^{(i)}(t)$ in order to incorporate the hypothesis dependence. This leads to the following simple modification to Eq. (6): 


$$
\lambda_{m n}^{(i)}(t)= \begin{cases}\lambda_{s, m n}(t)+\lambda_{b} & (i-1) \tau \leq t<i \tau \\ \lambda_{b} & \text { else }\end{cases}
$$

where $\lambda_{s, m n}(t)$ is the signal intensity function for the $m n$th detector element, often assumed to be constant over the $i$ th slot duration, and

$$
\lambda_{s}(t)=\sum_{m, n} \lambda_{s, m n}(t)
$$

is the total signal energy collected by the array. Suppose that each of the $M$ messages is equally likely to be transmitted with probability $M^{-1}$, and that each message generates a unique vector of detector array intensities at the receiver, denoted by $\boldsymbol{\lambda}^{(i)}(t)=\left(\lambda_{11}^{(i)}(t), \lambda_{12}^{(i)}(t), \cdots, \lambda_{K L}^{(i)}(t)\right)$. At the end of $T \mathrm{~s}$, the postdetection processor computes the probability of having received the observed array of count accumulator functions and selects that message corresponding to the greatest probability of having been received. Equivalently, the decoder selects the message corresponding to the greatest log-likelihood function, $\Lambda_{i}(T)$, evaluated after $T \mathrm{~s}$ and conditioned upon the signal occurring in the $i$ th time-slot:

$$
\begin{aligned}
\Lambda_{i}(T) & =\ln \left\{p\left[\mathbf{N}(t) \mid \boldsymbol{\lambda}^{(i)}(t) ; 0 \leq t<T\right]\right\}=\sum_{m=1}^{K} \sum_{n=1}^{L} \ln \left\{p\left[N_{m n}(t) \mid \lambda_{m n}^{(i)}(t) ; 0 \leq t<T\right]\right\} \\
& =\sum_{m=1}^{K} \sum_{n=1}^{L}\left(-\int_{(i-1) \tau}^{i \tau} \lambda_{m n}^{(i)}(t) d t+\sum_{w_{j, m n} \in((i-1) \tau, i \tau]} \ln \lambda_{m n}^{(i)}\left(w_{j, m n}\right)\right)+\left(\begin{array}{c}
\text { terms that depend } \\
\text { only on } \lambda_{b}
\end{array}\right)
\end{aligned}
$$

where $w_{j, m n}$ is the occurrence time of the $j$ th photon over the $m n$th detector element within the same time slot. Writing Eq. (7) over the $i$ th time slot as

$$
\lambda_{m n}^{(i)}(t)=\lambda_{b}\left(1+\frac{\lambda_{s, m n}(t)}{\lambda_{b}}\right), \quad(i-1) \tau \leq t<i \tau
$$

leads to the following useful logarithmic form:

$$
\ln \left(\lambda_{m n}^{(i)}(t)\right)=\ln \left(\lambda_{b}\right)+\ln \left(1+\frac{\lambda_{s, m n}(t)}{\lambda_{b}}\right)
$$

Assuming constant signal intensity over the $i$ th slot, independent of the value of $i$, substituting Eq. (11) into Eq. (9), and ignoring terms that depend only on $\lambda_{b}$ (hence do not convey any information about the transmitted symbol), the log-likelihood function reduces to

$$
\begin{aligned}
\Lambda_{i}(T) & =\sum_{m=1}^{K} \sum_{n=1}^{L}\left\{\sum_{w_{j, m n} \in((i-1) \tau, i \tau]} \ln \left(1+\frac{\lambda_{s, m n}\left(w_{j, m n}\right)}{\lambda_{b}}\right)\right\} \\
& =\sum_{m=1}^{K} \sum_{n=1}^{L} \ln \left(1+\frac{\lambda_{s, m n}}{\lambda_{b}}\right) N_{m n}^{(i)}
\end{aligned}
$$


where $N_{m n}^{(i)}$ is defined as the total number of photons occurring over the $m n$th detector element during the $i$ th time-slot. Note that with constant signal intensities the actual arrival times of photons within each slot do not contribute to the decision; hence, only the total number of detected photons, $N_{m n}^{(i)}$, matters. Given that we know the intensity over each detector element, the $i$ th log-likelihood function consists of the sum of a logarithmic function of the ratio of signal and background intensities from all detector elements over the $i$ th pulse interval, multiplied by the total number of detected photons; the optimum detection strategy is to select the symbol corresponding to the greatest log-likelihood function.

\section{Performance of the Optimum Detector-Array Receiver}

The probability of a correct decision is just the probability that the log-likelihood function associated with the transmitted symbol exceeds all other log-likelihood functions. Thus, when the $q$ th symbol was sent, then a correct decision is made if $\Lambda_{q}(T)>\Lambda_{i}(T)$ for all $i \neq q$. Denoting the logarithmic functions, or weights, in Eq. (12) by $u_{m n}$, the log-likelihood function can be rewritten as

$$
\Lambda_{i}(T)=\sum_{m=1}^{K} \sum_{n=1}^{L} u_{m, n} N_{m n}^{(i)}
$$

In this form, we can see that the log-likelihood function is composed of sums of a random number of weights from each detector element: for example, the $m n$th detector element contributes an integer number of its own weight to the sum. The probability density of the log-likelihood function is the convolution of the probability densities from each detector element, and, therefore, all possible combinations of sums are represented. This means that the probability masses in the convolved density are defined over all possible values the weights can assume (that is, all possible sums of weights from every detector element), and the probability over each point is the product of the individual probabilities contributing to that point. Clearly, the average values of the component probabilities depend on which hypothesis is actually true: if hypothesis $q$ is true, denoted by $H_{q}$, then the intensity in the $q$ th slot contains both signal and background energy, whereas the intensities in all other slots contain background energy only.

Define the set of ordered numbers over which the probability density of the $i$ th log-likelihood function takes on values as $\left\{\alpha_{0}=0, \alpha_{1}, \alpha_{2}, \cdots\right\}$ and let $\operatorname{Pr}\left[\Lambda_{i}(T)=\alpha_{k} \mid H_{q}\right] \equiv p_{i}\left(\alpha_{k} \mid H_{q}\right)$. Two distinct cases of interest arise, namely when $i=q$ and when $i \neq q$. With $H_{q}$ true, the intensities during the $q$ th time slot are governed by the intensity $\lambda_{s, m n}+\lambda_{b}$ over the $m n$th element of the detector array, whereas for every other nonsignal time slot the intensity over each detector element is simply $\lambda_{b}$. Note that the same weights are used when testing each slot, since the log-likelihood function associated with a given slot assumes that the signal really is in that slot; therefore, the domain of the convolved density does not depend on the hypothesis, although the resulting probabilities defined over each point do.

The received symbol is decoded correctly if the sum of weights from all detector elements over the signal slot exceed the sum of weights from every other (nonsignal) slot. However, since the weights for each log-likelihood function are the same, it is possible that the maximum sum of weights occurs over two or more slots, one of which is the true signal slot, thus creating a "tie for biggest." This tie is optimally resolved by a random choice among the biggest likelihood functions: if there are $r$ nonsignal slots tied for biggest with the correct signal slot, then the probability of selecting the correct symbol in the presence of $(r+1)$ ties for biggest is $(r+1)^{-1}$. However, there are " $(M-1)$ taken $r$ at a time" ways these ties can occur among $M$ slots. On the other hand, if none of the detectors registers a photon over any of the $M$ slots, then we have to make a random choice among $M$ possibilities, which yields a correct decision with probability $M^{-1}$. As in [2], taking all cases into account and assuming equiprobable signals, the probability of correctly decoding the received symbol is 


$$
\begin{aligned}
P_{M}(C)= & P_{M}\left(C \mid H_{q}\right) \\
= & \left\{\sum_{r=0}^{M-1}\left(\frac{1}{r+1}\right)\left(\begin{array}{c}
M-1 \\
r
\end{array}\right) \sum_{k=1}^{\infty} p_{q}\left(\alpha_{k} \mid H_{q}\right) \underset{\substack{i \neq q \\
i \neq}}{\left.\left(\alpha_{k} \mid H_{q}\right)\right]^{r}\left[\sum_{j=0}^{k-1} p_{i}\left(\alpha_{j} \mid H_{q}\right)\right]^{M-1-r}}\right\} \\
& +M^{-1}\left\{p_{q}\left(\alpha_{0} \mid H_{q}\right)\left[p_{i}\left(\alpha_{0} \mid H_{q}\right)\right]^{M-1}\right\}
\end{aligned}
$$

where $p_{q}$ and $p_{i}, i \neq q$, refer to the probability densities corresponding to the signal and non-signal hypotheses, respectively. The probability of a symbol error is simply $P_{M}(E)=1-P_{M}(C)$. When the array consists of a single detector, the weights can be set to one, and the probabilities then are governed by the Poisson density defined over the integers.

Note that by counting all ties as errors in Eq. (14), a lower bound on the probability of correct detection, $P_{M}^{l}(C)$, is obtained that is much easier to compute, namely,

$$
P_{M}(C) \geq P_{M}^{l}(C) \equiv \sum_{k=1}^{\infty} p_{q}\left(\alpha_{k} \mid H_{q}\right)\left[\sum_{j=0}^{k-1} p_{i}\left(\alpha_{j} \mid H_{q}\right)\right]^{M-1}+M^{-1}\left\{p_{q}\left(\alpha_{0} \mid H_{q}\right)\left[p_{i}\left(\alpha_{0} \mid H_{q}\right)\right]^{M-1}\right\}
$$

This lower bound on the probability of correct detection yields the following useful upper bound on the error probability:

$$
P_{M}^{u}(E) \equiv 1-P_{M}^{l}(C) \geq P_{M}(E)
$$

This upper bound will be evaluated in the following sections for several cases of interest.

\section{E. The Adaptive Synthesized Detector Receiver}

We observe from the preceding analysis that detectors containing much more background than signal intensity do not contribute significantly to the error probability, since the output of these detector elements is multiplied by weights that are close to zero. This observation suggests the following suboptimum decoder concept with greatly simplified structure: list the detector elements starting with the one containing the most signal energy and followed by every other detector ordered according to decreasing signal intensities. Compute the probability of error for the first detector element plus background; then form the sum of signal energies from the first two detector elements (plus background for two detector elements), and so on, until the minimum error probability is reached. Each set of detectors may be considered to be a single detector, so that no weighting is applied to account for variations in the signal distribution over the detector elements included in that set. The set of detector elements that achieves the minimum probability of error is the best synthesized single detector matched to the signal-intensity distribution. However, this straightforward process of performing the optimization by actually calculating the error probabilities for each partial sum of detectors is not practical. Later in this article, we shall describe some practical methods for approximating this procedure. 
In effect, we have partitioned the logarithmic weights into two classes: large weights were assigned the value one, while small weights were assigned the value zero. We shall show that this simple partitioning achieves near-optimum performance in low to moderate background environments, but with greatly reduced decoder complexity.

For the adaptive synthesized single detector, the probability of correct decision can be obtained directly from Eq. (14) by setting $\alpha_{k}=k$ in the probability densities and assuming constant signal and background intensities over each time slot, yielding

$$
\left.\begin{array}{l}
p_{q}\left(k \mid H_{q}\right)=\frac{\left(\lambda_{s} \tau+\lambda_{b} \tau\right)^{k}}{k !} e^{-\left(\lambda_{s} \tau+\lambda_{b} \tau\right)} \\
p_{i}\left(k \mid H_{q}\right)=\frac{\left(\lambda_{b} \tau\right)^{k}}{k !} e^{-\lambda_{b} \tau}
\end{array}\right\}
$$

Direct substitution of these Poisson densities into Eq. (14) yields

$$
\begin{aligned}
& P_{M}(C)= \\
& \quad\left\{\sum_{r=0}^{M-1}\left(\frac{1}{r+1}\right)\left(\begin{array}{c}
M-1 \\
r
\end{array}\right) \sum_{k=1}^{\infty} \frac{\left(\lambda_{s} \tau+\lambda_{b} \tau\right)^{k}}{k !} e^{-\left(\lambda_{s} \tau+\lambda_{b} \tau\right)}\left[\frac{\left(\lambda_{b} \tau\right)^{k}}{k !} e^{-\lambda_{b} \tau}\right]^{r}\left[\sum_{j=0}^{k-1} \frac{\left(\lambda_{b} \tau\right)^{j}}{j !} e^{-\lambda_{b} \tau}\right]^{M-1-r}\right\} \\
& \quad+M^{-1} e^{-\left(\lambda_{s}+M \lambda_{b}\right) \tau}
\end{aligned}
$$

where again $P_{M}(E)=1-P_{M}(C)$.

\section{F. The Gaussian Approximation}

When the array contains a large number of detector elements, the computation of the probability density of the weighted sum of Poisson random variables becomes prohibitively difficult. In that case, approximating the true discrete density with a simpler continuous density would be of great value. It is shown in the Appendix that a useful Gaussian approximation to the discrete density of the weighted sum of Poisson random variables can indeed be derived from the characteristic function of the discrete density. The mean and variance of the approximating continuous random variable, $\eta$ and $\sigma^{2}$, respectively, are defined as

$$
\begin{gathered}
\eta=\sum_{m=1}^{K} \sum_{n=1}^{L} u_{m n} \lambda_{m n} \tau \\
\sigma^{2}=\sum_{m=1}^{K} \sum_{n=1}^{L} u_{m n}^{2} \lambda_{m n} \tau
\end{gathered}
$$

We expect this approximation to be accurate when either of two conditions is satisfied: when the average photon energies over each detector element are so high that the Poisson distribution can be well approximated by a Gaussian density (this would occur with intense background radiation) or when the conditions for the central limit theorem are satisfied. The central limit theorem applies when the average 
photon energies over the entire array are sufficiently similar that a large number of detector outputs can be considered identically distributed random variables or when the array outputs can be partitioned into several groups of random variables, each containing a large enough number of random variables to justify the Gaussian model. With this approximation, the discrete convolved density of weighted Poisson random variables can be replaced by a continuous Gaussian random variable, with mean and variance as defined above.

Assuming equal a priori symbol probabilities, the probability of correctly decoding the received PPM symbol is again the probability that the random variable in the signal-plus-noise time slot exceeds all of the other random variables in the remaining $(M-1)$ noise-only time slots (we need not consider equalities here, since with continuous random variables equality is a zero-probability event). Recalling that the average intensity in the signal slot is $\left(\lambda_{s, m n}+\lambda_{b}\right) \tau$ while in all of the other slots it is $\lambda_{b} \tau$, and defining the means and variances associated with signal and nonsignal slots in Eq. (14) as $\eta_{s b}, \sigma_{s b}^{2}$ and $\eta_{b}, \sigma_{b}^{2}$, respectively, the probability of a correct decision takes the form

$$
P_{M}(C) \cong \int_{-\infty}^{\infty} d y \frac{e^{-\left(y-\eta_{s b}\right)^{2} / 2 \sigma_{s b}^{2}}}{\sqrt{2 \pi \sigma_{s b}^{2}}}\left\{\int_{-\infty}^{y} d x \frac{e^{-\left(x-\eta_{b}\right)^{2} / 2 \sigma_{b}^{2}}}{\sqrt{2 \pi \sigma_{b}^{2}}}\right\}^{(M-1)}
$$

After some manipulation, the probability of symbol error can be put into the form

$$
P_{M}(E)=1-P_{M}(C) \cong 1-\int_{-\infty}^{\infty} d y \frac{e^{-y^{2} / 2}}{\sqrt{2 \pi}}\left\{1-\frac{1}{2} \operatorname{erfc}\left(\frac{\sigma_{s b}}{\sigma_{b}} y+\frac{\eta_{s b}-\eta_{b}}{\sigma_{b}}\right)\right\}^{(M-1)}
$$

where erfc is the complementary error function defined as $\operatorname{erfc}(x)=(1 / \sqrt{2 \pi}) \int_{x}^{\infty} e^{-y^{2} / 2} d y$.

\section{G. Performance Bounds for Poisson Detection}

For the special case of binary PPM signaling, $M=2$, the following useful form has been obtained by Hubbard [5]:

$$
P_{2}(E)=\exp \left(-\Delta^{2}\right)\left\{\sum_{k=0}^{\infty}\left(\frac{\sqrt{\lambda_{b} \tau}}{\Delta+\sqrt{\lambda_{b} \tau}}\right)^{k} F_{k}(\psi)-\frac{1}{2} F_{0}(\psi)\right\}
$$

where $\Delta=\sqrt{\left(\lambda_{s}+\lambda_{b}\right) \tau}-\sqrt{\lambda_{b} \tau}, \psi=2 \tau \sqrt{\left(\lambda_{s}+\lambda_{b}\right) \lambda_{b}}$, and $F_{k}(\psi)=e^{-\psi} I_{k}(\psi)$, where $I_{k}(\psi)$ is the modified Bessel function of order $k$.

Since for any $\psi \geq 0$ and $k \geq 0, I_{k}(\psi) \leq I_{0}(\psi)$, a useful upper bound to Eq. (22) can easily be constructed as

$$
P_{2}(E) \leq \exp \left(-\Delta^{2}\right)\left\{\sum_{k=0}^{\infty}\left(\frac{\sqrt{\lambda_{b} \tau}}{\Delta+\sqrt{\lambda_{b} \tau}}\right)^{k}-\frac{1}{2}\right\} F_{0}(\psi)=\exp \left(-\Delta^{2}\right)\left(\frac{\sqrt{\lambda_{b} \tau}}{\Delta}+\frac{1}{2}\right) F_{0}(\psi) \equiv P_{2}(U B)
$$

This upper bound on the binary PPM error probability can be further approximated in the limit of very small and very large background energies as 


$$
P_{2}(U B) \cong \begin{cases}\frac{1}{2} \exp \left(-\Delta^{2}\right) & \lambda_{b} \tau<<1 \\ \left(\frac{\sqrt{\lambda_{b} \tau}}{\Delta}+\frac{1}{2}\right) \frac{\exp \left(-\Delta^{2}\right)}{\sqrt{2 \pi \psi}} & \lambda_{b} \tau>>1\end{cases}
$$

The binary PPM error probability and its upper bound also can be used to further bound Mary PPM performance for the single-detector case, in a manner similar to the union bound familiar from the additive Gaussian noise problem. The result is an expression of the form

$$
P_{M}(E) \leq(M-1) P_{2}(E)
$$

Proof of this is somewhat complicated by the fact that, for the optical problem, the probability of committing an error when attempting to resolve ties among maximal counts also must be taken into account. The proof is presented in $[6$, Appendix B].

\section{H. Comparison of Exact and Approximate Performance Calculations}

A performance comparison of the optimally weighted array receiver and the adaptive synthesized single-detector receiver (also referred to as the 0-1 subarray in the figures) has been carried out for average background energies of $K_{b} \equiv \lambda_{b} \tau=0.1$ and 1.0. Two different signal models were used: a simple test model wherein only 5 of the $16 \times 16=256$ total detector elements were assumed to contain signal energy while the rest were assumed to contain no signal, and a more realistic $16 \times 16$ detector array model wherein the signal distribution over the array was simulated using a Kolmogorov turbulence model as described in [7] and all 256 detector elements may contain some signal.

For the test model, the proportions of the total average absorbed signal energy, $K_{s} \equiv \lambda_{s} \tau$, over the five detector elements were assumed to be $(1.0,0.3,0.2,0.05,0.02)$. Equations (15) and (16) were evaluated using this model and compared with results obtained via Monte Carlo simulations. The results are shown in Fig. 3 as a function of the total average absorbed signal energy, $K_{s}$. It is evident that optimal weighting

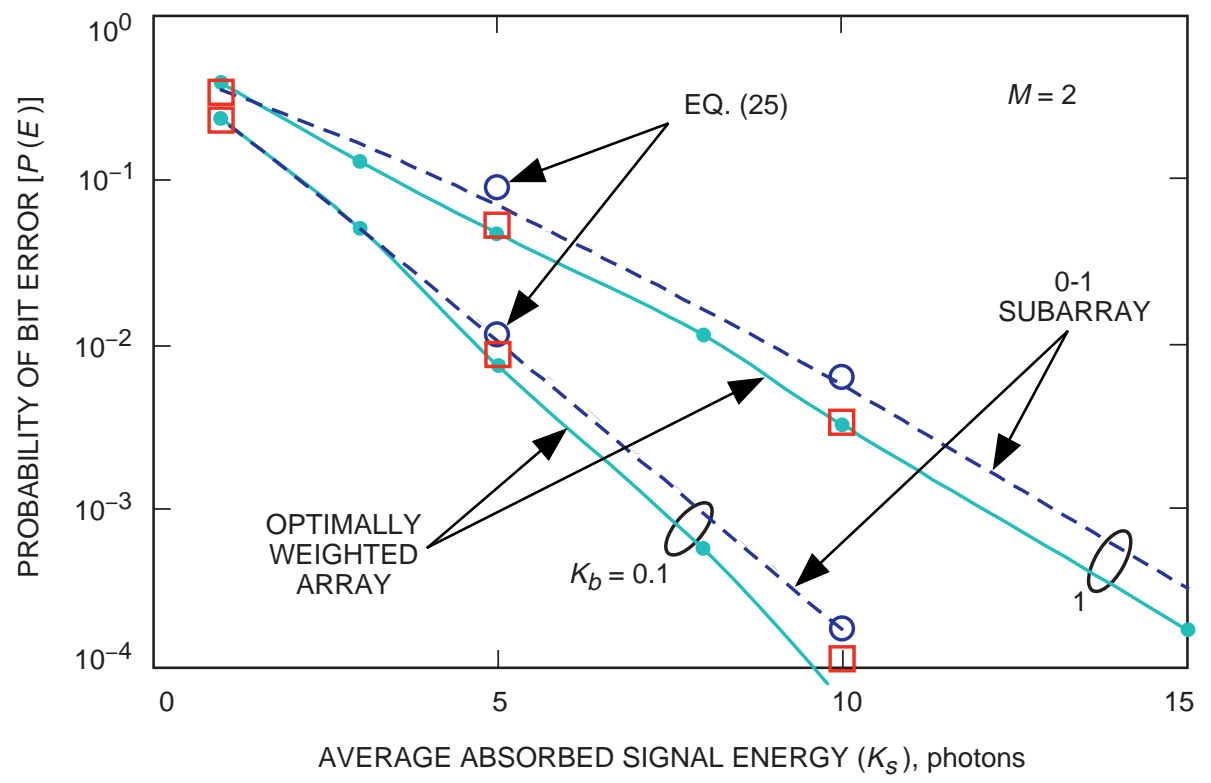

Fig. 3. The performance of the optimally weighted array and the adaptive 0-1 array, binary PPM. 
(red squares represent computed and light blue lines simulated data) yields somewhat better performance than the suboptimum 0-1 subarray computed according to Eq. (18), and that greater improvements occur at greater background intensities; however, the improvements due to the significantly more complicated optimally weighted array are only about $0.3 \mathrm{~dB}$ at an error probability of 0.001 for the high background case.

The reason for using only five detectors in the examples of Fig. 3 is that convolving more than five weighted Poisson densities rapidly becomes prohibitively difficult, due to excessive demands on computer memory. Therefore, only this five-detector example could be evaluated computationally through the use of the bound on the probability of correct detection defined in Eq. (15), and only for background intensities not exceeding an average of 2 photons per detector per slot. Note the excellent agreement between the calculated error probabilities for the 0-1 subarray case and the approximate upper bound of Eq. (25), represented by the large blue circles in Fig. 3 at average signal energies of 5 and 10 photons.

In Fig. 4, a realistic spatial distribution of the signal intensity over the focal plane was generated using Kolmogorov phase screens. Monte Carlo simulations were performed to evaluate the error probability for the optimally weighted array. The Gaussian approximation to the error probability defined in Eq. (20) also has been evaluated for the binary PPM case, $M=2$, with average background energies (per detector element) of $K_{b} \equiv \lambda_{b} \tau$ equal to $0.1,1$, and 5 photons/time slot, as a function of the total average signal energy. It can be seen that the Gaussian approximation is close to the exact values obtained from Monte Carlo simulation and that good agreement is obtained even for small background energies per detector element, as direct comparison with the simulation results (large diamonds) indicates. In fact, it appears that this Gaussian approximation provides useful results over the entire range of background and signal energies represented in Fig. 4. The performance of the 0-1 subarray also is shown, as the blue dashed curves; as expected, its performance is somewhat worse than that of the optimally weighted array, but not significantly so.

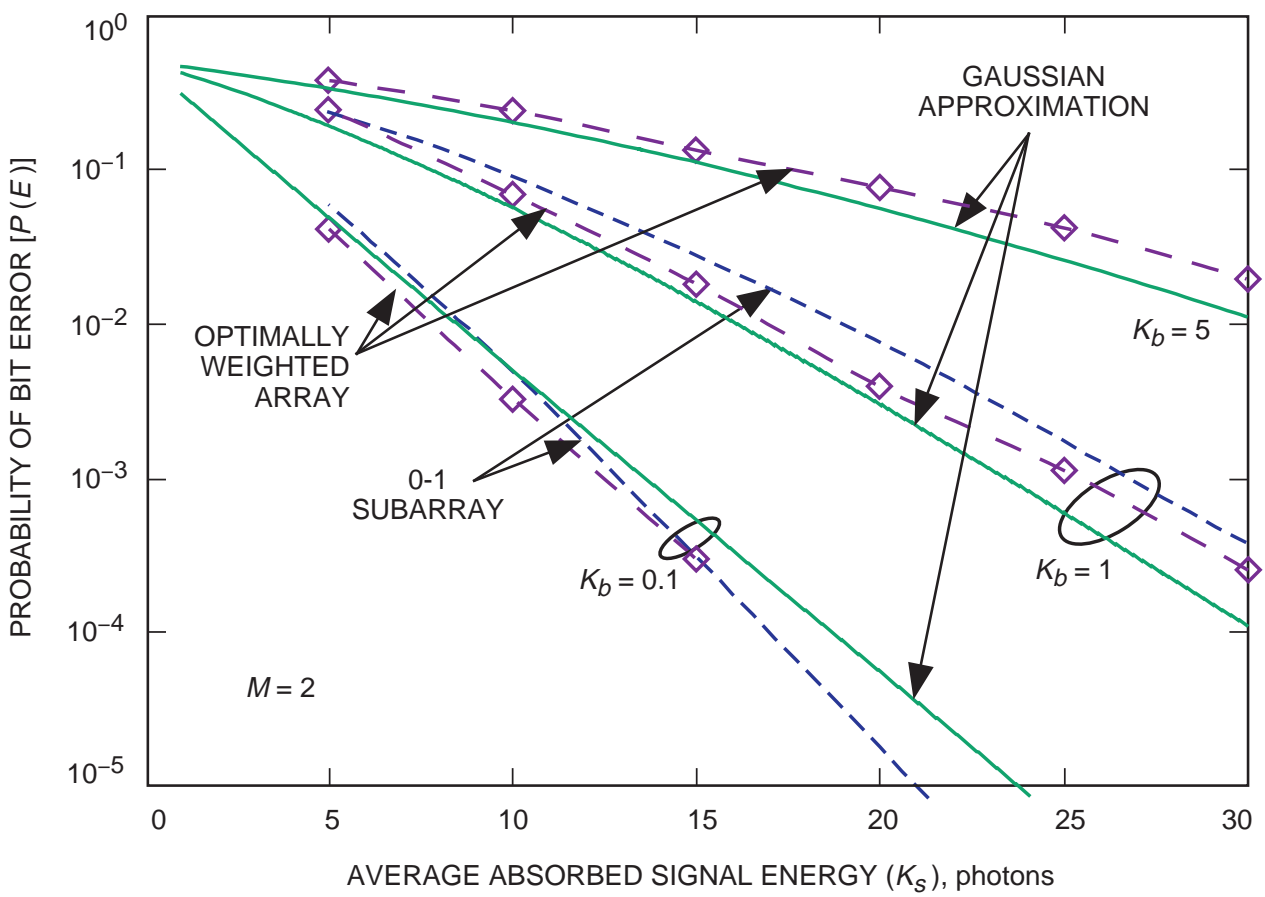

Fig. 4. The exact error probabilities, Gaussian approximations to the weighted sum, and simulation results for the realistic signal distribution described in Section III. 


\section{Numerical Results}

\section{A. Performance of an Optimally Weighted Array}

Both analytical calculations and Monte Carlo simulations were performed in order to obtain PPM error probabilities for the adaptive synthesized single-detector subarray. Performance of the optimally weighted array receiver was obtained from simulations; for each PPM symbol, $M$ Poisson random variables with the proper statistics were generated, the optimum weights were applied, and the symbol corresponding to the largest observable selected. Simulated turbulence-degraded signal distributions were generated over the $16 \times 16$ detector array for all subsequent results. With no loss in generality, the transmitted symbol always was assumed to be the one corresponding to a signal pulse in the first slot. The detection process was repeated a large number of times (until 100 errors were accumulated) and repeated for increasing average signal energy with various background levels. The results of this simulation are shown in Figs. 5(a) and 5(b) for $M=2,16$, and 256. The probability of bit error is shown as a function of the receiver's photon efficiency, $\rho$, which is a measure of the average number of bits of information carried by each photon. It can be seen that, with background levels from 0.1 to 1.0 photons per slot, $\rho$ from 0.3 to 0.5 bits/photon can be achieved with 256 PPM signaling at uncoded symbol-error probabilities around 0.001 to 0.01 .

\section{B. Performance of Adaptive Synthesized Single-Detector Receiver}

In order to generate a spatial distribution of the signal incident upon the detector plane, a sample field was generated using a Kolmogorov phase-screen program [7], resulting in a matrix of complex signal amplitudes. For the simulation, an atmospheric correlation length of $r_{0}=4 \mathrm{~cm}$ was assumed, which
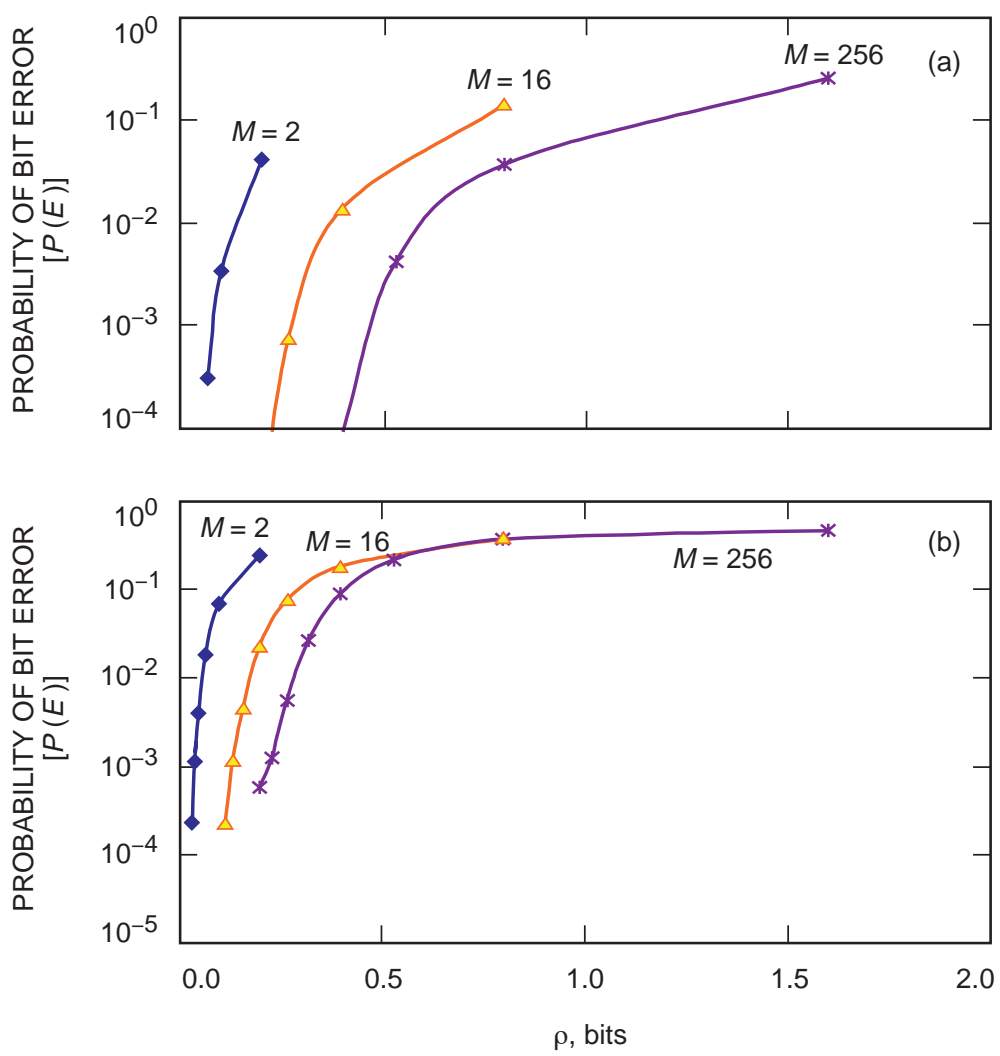

Fig. 5. The simulated PPM bit-error probability of the optimum array receiver as a function of photon efficiency, $\rho$ bits/photon: (a) $K_{b}=0.1$ and (b) $K_{b}=1.0$. 
implies that the results should apply to any receiving aperture that is much greater than this correlation length [1]. The field intensity generated in the detector plane by the simulation then is integrated over the elements of a $16 \times 16$ detector array, which is assumed to encompass the extent of the signal distribution in the detector plane. The detector signal intensities are normalized so that for the $m n$th detector we obtain an average number of absorbed signal photons of $\lambda_{s, m n} \tau$. A constant average background photon energy of $\lambda_{b} \tau$ is assumed over each detector element.

For a given sample function of the intensity distribution, the $16 \times 16=256$ detector elements were sorted in decreasing order of average signal energy, and Mary PPM symbol-error probabilities were calculated for increasing numbers of detectors, starting with the first detector, using Eq. (15). Figure 6 shows the symbol-error probability for binary PPM, $M=2$, as a function of the number of detector elements used for the case $K_{s}=10$ and $K_{b}=0.1$ (that is, the total average signal photons absorbed by the entire array is 10 and the average number of background photons per detector element is 0.1 ). It can be seen that, for this case, the smallest error probability of 0.0049 is achieved by assigning unity weight to the first 15 detector elements containing the greatest signal intensities and zero to all the rest.

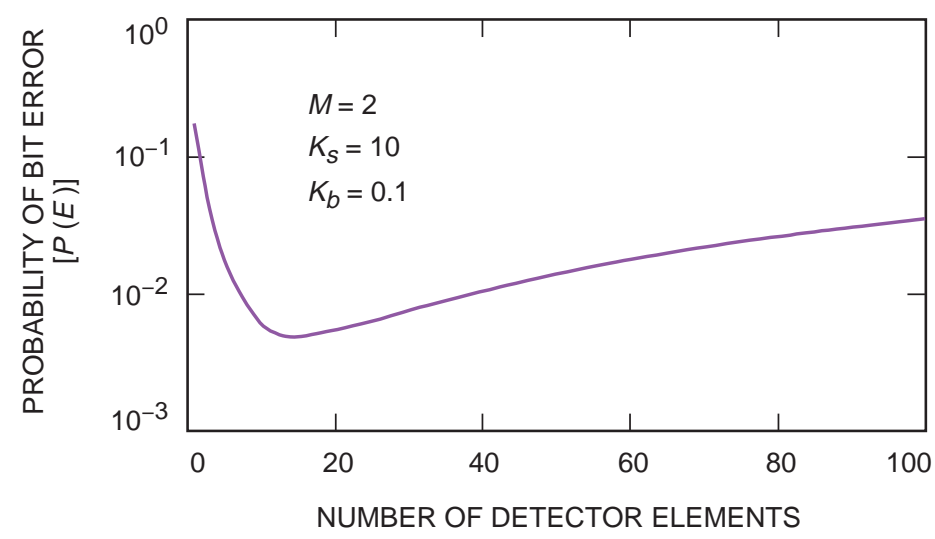

Fig. 6. The probability of error as a function of the number of sorted detector elements.

In Figs. 7(a) and 7(b), binary PPM symbol-error probabilities are shown as a function of total average number of absorbed signal photons for four cases: (1) when the optimum number of unweighted detector elements is used, (2) when the optimally weighted array is simulated, (3) when all 256 detector elements are given unity weight (synthesizing a large, nonadaptive single-detector element), and (4) when an ideal adaptive optics system succeeds in concentrating all of the available signal energy into a single detector element, which then is the only detector element that is observed.

Using the same focal-plane signal distribution as before, error probabilities were computed for average background photon counts of 0.1 and 1.0, shown in Figs. 7(a) and 7(b), indicating performance gains by the adaptive synthesized single detector over a single large nonadaptive detector of 2 and 2.8, respectively, at an error probability of 0.001 , corresponding to 3 and $4.5 \mathrm{~dB}$ of performance improvement. Note the excellent agreement of the approximation of Eq. (24) (large blue circles) with the computed values. When compared with the ideal adaptive optics receiver that concentrates all of the collected signal energy in a single element of the array, the gains are 3.8 and 8.2, corresponding to 5.9 and $9.1 \mathrm{~dB}$ of improvement. Note that the optimal weighted array yields only about $0.3 \mathrm{~dB}$ of improvement over the optimized $0-1$ subarray at a symbol-error probability of 0.001 , even with a relatively high background energy of $K_{b}=1$.

Similar gains are evident in Figs. 7(c) and 7(d), which represent the symbol-error probability, $P(S E)$, of the optimized subarray observing 16-dimensional $(M=16)$ PPM. The accuracy of the union bound of Eq. (26) evaluated for the case $\lambda_{b} \tau>>1$ is evident (large blue circles), especially at the lower error probabilities. 

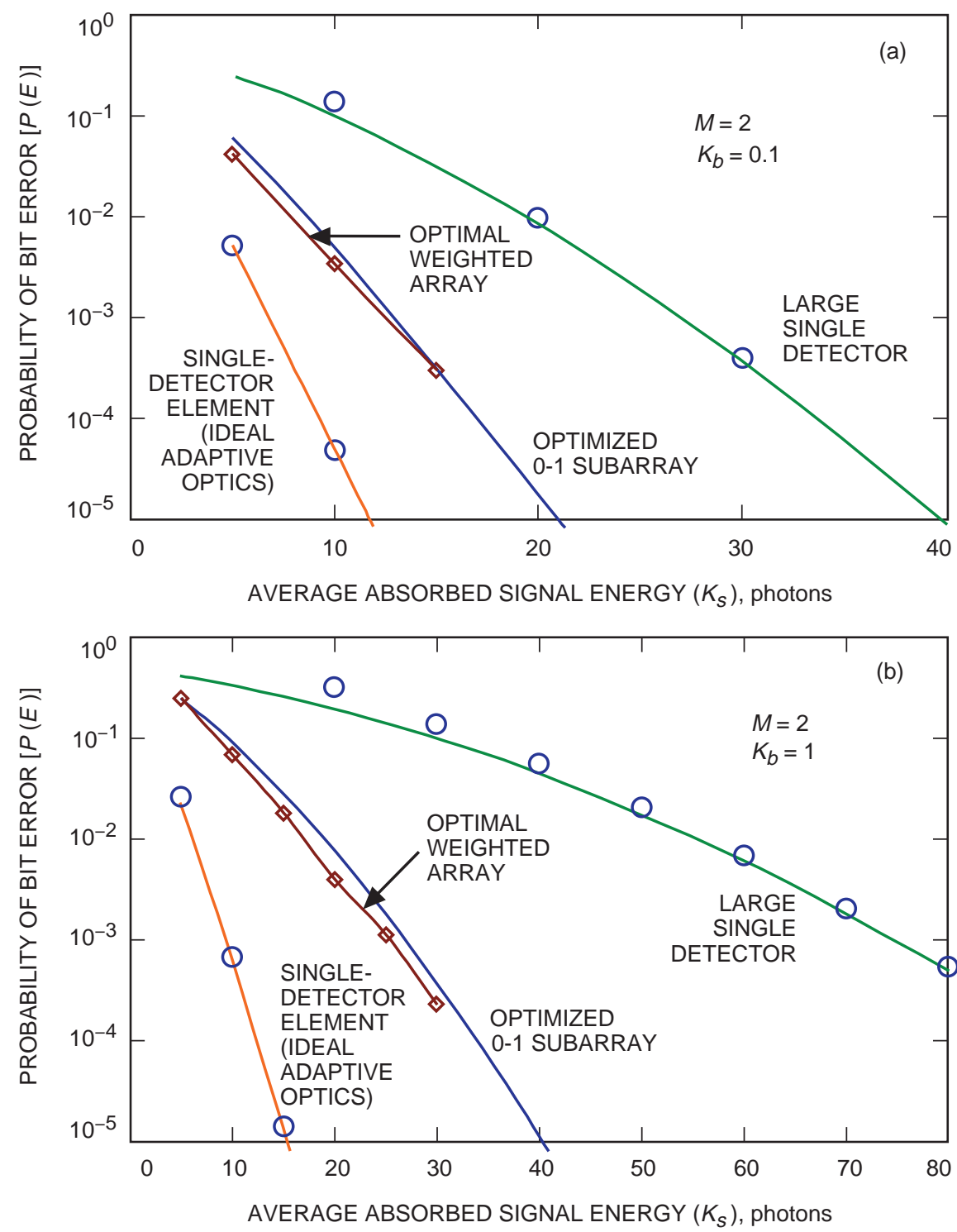

Fig. 7. The binary error probability of the large single detector, optimally weighted array, optimized 0-1 subarray, and single-detector element with ideal adaptive optics for (a) $K_{b}=0.1$ and (b) $K_{b}=1$, and the receiver performance for $M=16$ for (c) $K_{b}=0.1$ and (d) $K_{b}=1$.

Performance improvements also were obtained for several different focal-plane distributions at an average background energy of one photon per detector per slot to verify that the above results were typical. The results are shown in Fig. 8, where it can be seen that three out of four simulations yielded performance comparable to that of Fig. 7(b), requiring approximately 26 signal photons to achieve an error probability of 0.001 while utilizing 9 to 13 elements of the array in the region of error probabilities examined. One of the phase-screen simulations yielded an unusually favorable signal distribution that achieved optimum performance with only 4 detector elements but still needed roughly 22 signal photons for 0.001 error probability. We can conclude, therefore, that, for different distributions generated using the same atmospheric and receiver parameters, similar receiver performance is obtained; hence, it is reasonable to use a representative signal distribution for the subsequent numerical analysis. 

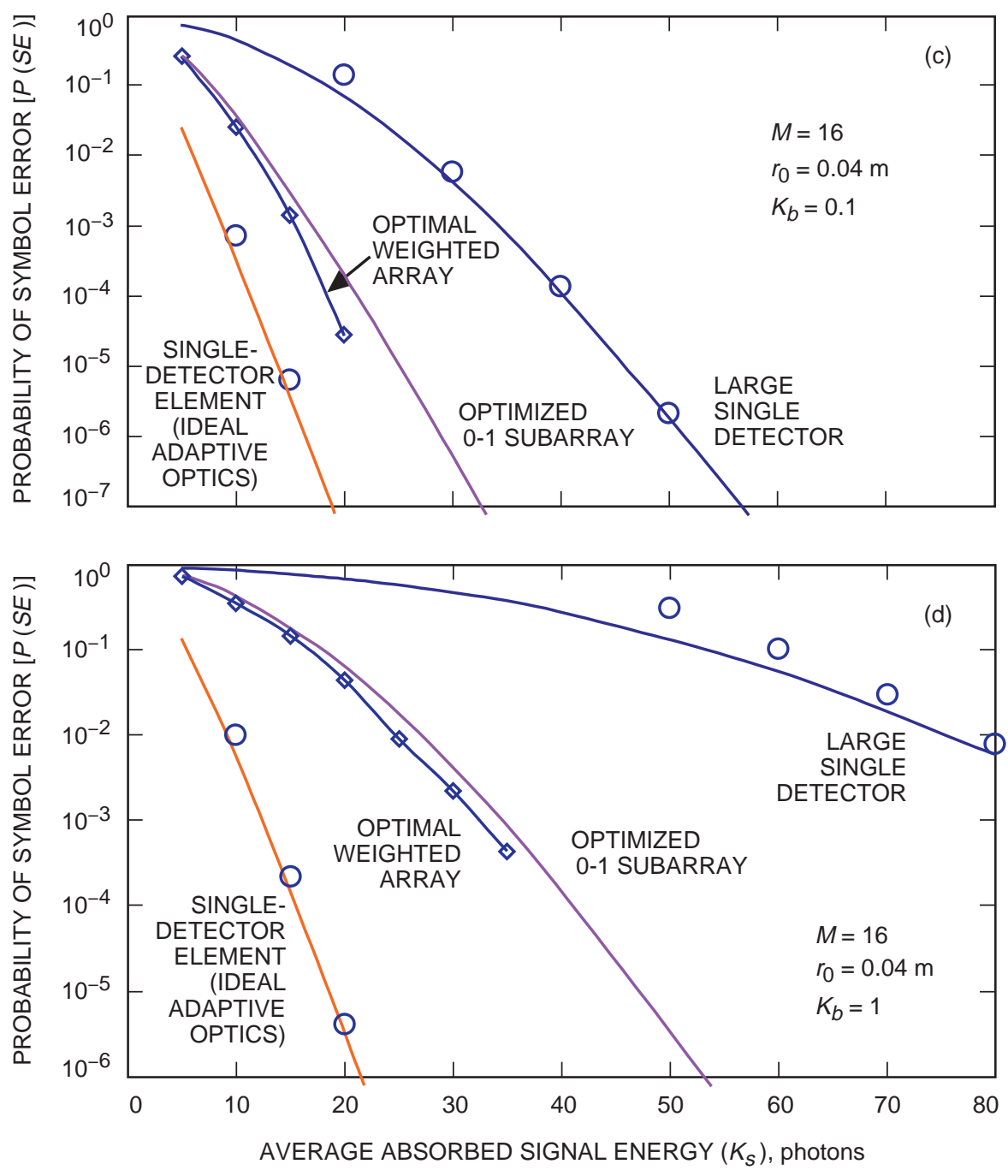

Fig. 7 (cont'd). The binary error probability of the large single detector, optimally weighted array, optimized $0-1$ subarray, and single-detector element with ideal adaptive optics for (a) $K_{b}=0.1$ and (b) $K_{b}=1$, and the receiver performance for $M=16$ for (c) $K_{b}=0.1$ and (d) $K_{b}=1$.

Note that as more detector elements are used the performance tends to deteriorate, since a greater amount of background energy is collected by the receiver. We shall continue with the original distribution that required 15 detector elements for optimum performance, corresponding to a slightly pessimistic signal distribution and implying that the performance improvements obtained with this distribution tend to be somewhat conservative.

\section{Algorithmic Optimization of the Number of Detector Elements}

One problem with the above approach for determining the optimum number of detector elements is that computing the error probability for each increasing subarray requires a great deal of computation time, particularly when large detector arrays are used; this may not be feasible in an operational system. As an alternative, we attempt to construct a numerical measure that takes into account the number of detectors and the total average signal and background energies to see if simpler computations would suffice for determining the optimum number of detector elements (that is, the number yielding the lowest error probability). Three different measures were constructed and evaluated, each bearing some resemblance 


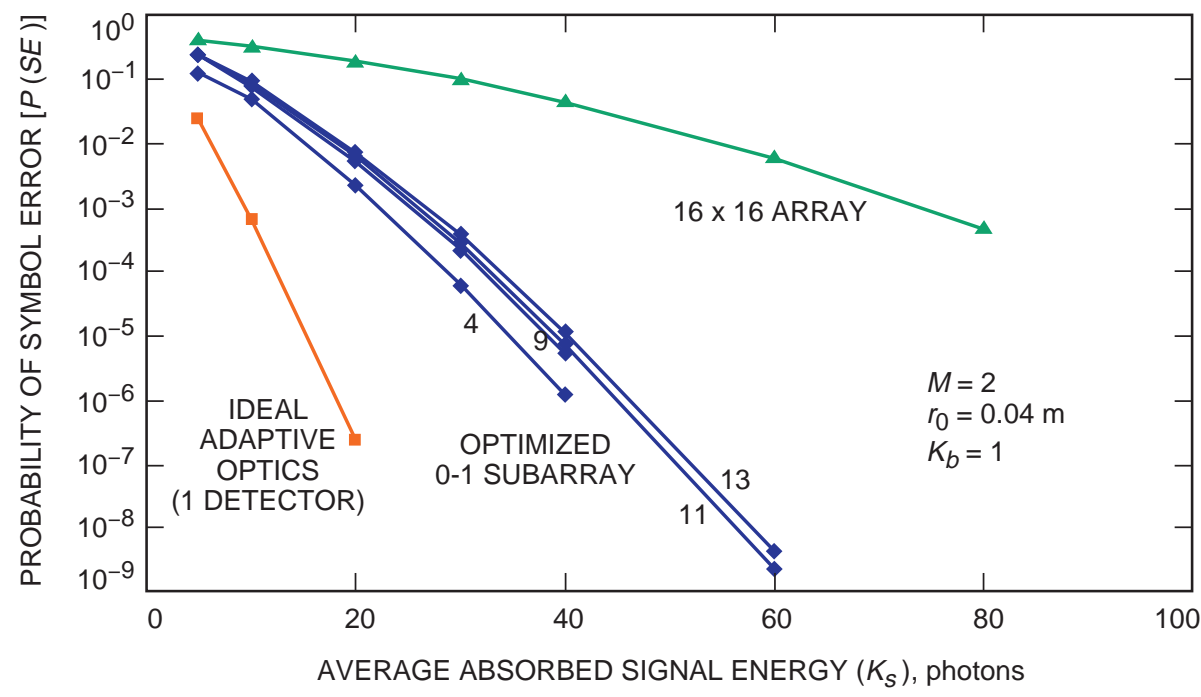

Fig. 8. The performance of the optimized 0-1 subarray with different turbulent signal distributions.

to a signal-to-noise ratio (SNR), although we realize that, unlike the case of additive Gaussian noise problems, in optical communications SNR generally is not a useful measure of performance. These three "easily computable" functions are defined as

$$
\begin{gathered}
\operatorname{SNR}_{1}(l)=\frac{\left(\sum_{i=1}^{l} \lambda_{s, m n} \tau\right)^{2}}{l \lambda_{b} \tau} \\
\operatorname{SNR}_{2}(l)=\frac{\left(\sum_{i=1}^{l} \lambda_{s, m n} \tau\right)^{2}}{l \lambda_{b} \tau+\sum_{i=1}^{l} \lambda_{s, m n} \tau}
\end{gathered}
$$

and

$$
\operatorname{SNR}_{3}(l)=\sqrt{\frac{4 \sum_{i=1}^{l} \lambda_{s, m n} \tau}{2 l \lambda_{b} \tau+1}}
$$

where the index $l$ represents the number of detector elements over which these functions are maximized, $1 \leq l \leq 256$ for the current example. Figure 9 shows the three functions versus $l$ for the case $\lambda_{s} \tau=10$ and $\lambda_{b} \tau=0.1$, assuming Poisson statistics. It can be seen that the maximum values for these three measures are reached when $l$ equals 10, 22, and 7 detector elements, resulting in binary PPM symbolerror probabilities of $5.978 \times 10^{-3}, 5.84 \times 10^{-3}$, and $1.049 \times 10^{-2}$, respectively, as compared with the performance of the true optimum subarray of $4.884 \times 10^{-3}$ calculated from the actual error probabilities and achieved with 15 detector elements. Figure 10 shows how subarrays obtained using the three different SNR measures compare with the performance of the true optimized subarray over a range of signal energies for a particular signal distribution. We can conclude that both $\mathrm{SNR}_{1}$ and $\mathrm{SNR}_{2}$ yield performances comparable to that of the optimized subarray and, therefore, could be used to optimize the subarray dimensions in real time; however, $\mathrm{SNR}_{3}$ provides a very poor estimate and hence should not be used. 


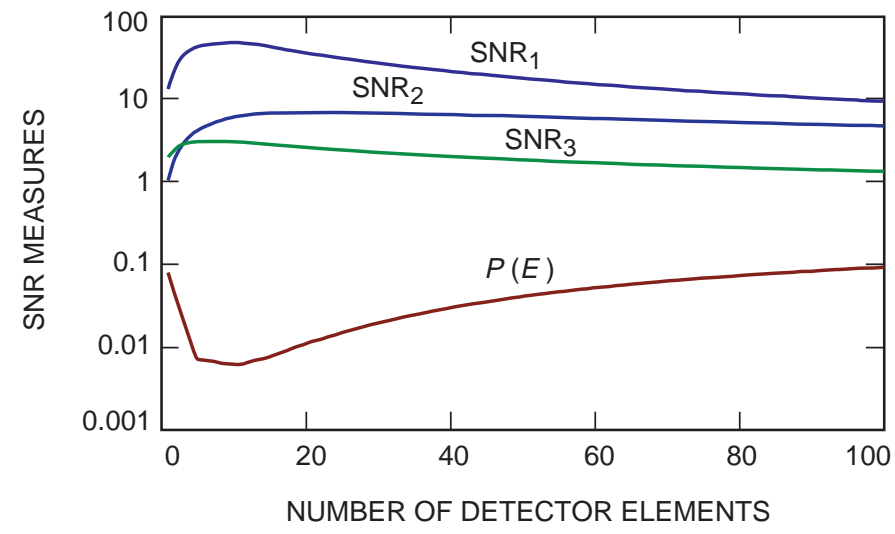

Fig. 9. Comparison of the optimum number of detector elements predicted by the three SNR measures.

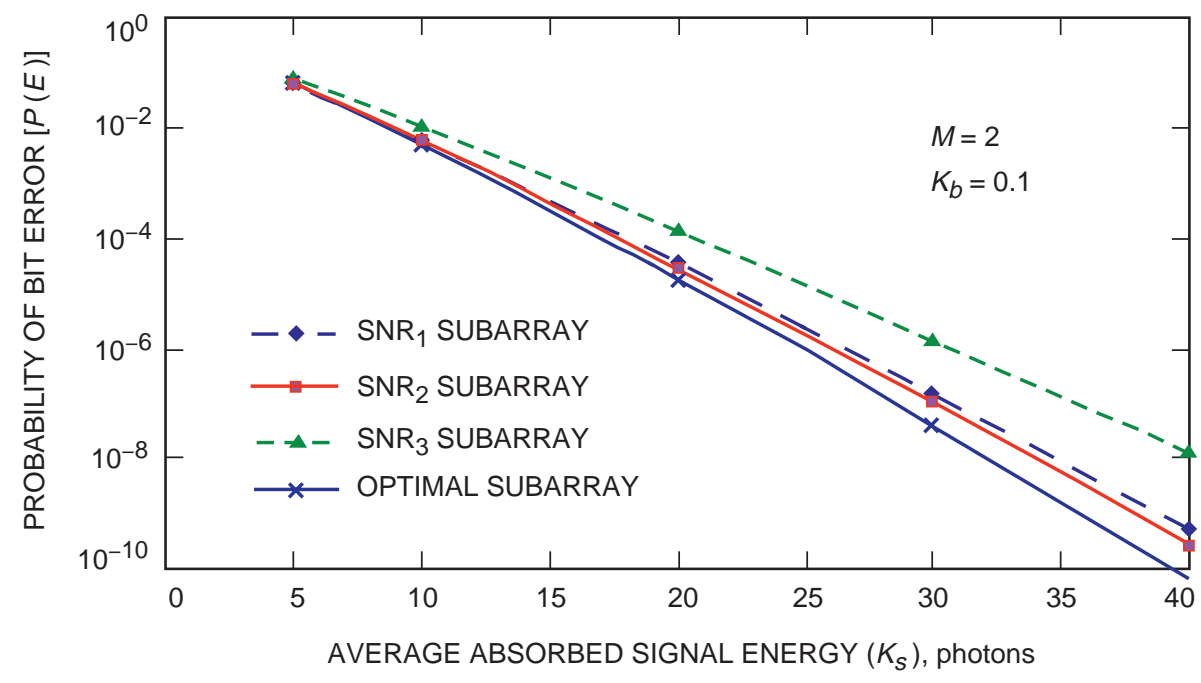

Fig. 10. Comparison of the three different SNR measures.

\section{Estimation of Signal Intensities}

Finally, we note that the numerical results presented thus far were obtained under the assumption that we know the true value of the average signal and background photons absorbed by each detector element, and, therefore, the sorting of the detector elements is based upon the true signal energies. However, the signal energies change with time due to turbulence, although the background intensity can be considered constant in most applications. Therefore, we examined the case when the signal energies were not known a priori but had to be estimated from the observed detector outputs. The results of simulations in which actual Poisson deviates were generated for each array element, and the mean signal energies estimated from the observed outputs, are presented in Fig. 11 for binary PPM.

For each detector array element, Poisson random variables were generated for the $M$ ary signal and background slots with average intensities obtained from the Kolmogorov phase-screen output plus a specified level of background light. These deviates were generated for $L$ PPM symbols and the slot outputs added for each detector element, resulting in $m \times n$ statistics: 


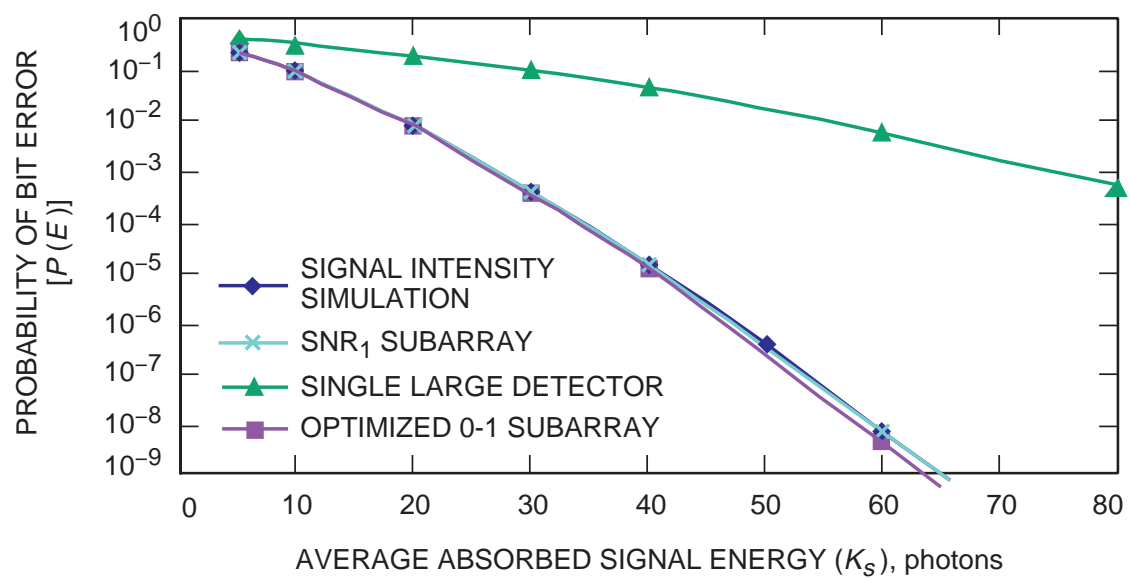

Fig. 11. Comparison of simulated and exact signal intensities.

$$
Y_{m n}=\sum_{i=1}^{L} \sum_{j=0}^{M-1} X_{m n}(i, j)
$$

where $X_{m n}(i, j)$ is the output of the $j$ th slot of the $i$ th PPM symbol. These statistics then were sorted as before from largest to smallest. The average number of signal photons was estimated from these statistics as

$$
\hat{\lambda}_{s, m n} \tau=\max \left(\frac{Y_{m n}}{L}-M \lambda_{b} \tau, 0\right)
$$

where we tacitly assumed that the actual background intensity can be estimated accurately, both because it is essentially constant in the detector plane and because typically there is significant dead time between PPM symbols to allow for transmitter laser recovery, which can be used to estimate the background intensity since no signal photons are present. Once the average signal and background energies have been estimated, $\mathrm{SNR}_{1}(l)$ may again be maximized over the number of elements, $l$, as before in order to obtain the optimum detector subarray.

Results for binary PPM with estimated signal energies are shown in Fig. 11 for an average background energy of 1.0 photon per detector element per slot. This figure shows the symbol-error probability as a function of total average absorbed signal energy, $K_{s}$, using estimates obtained from the simulation with $L=1000$, as well as three other curves where exact knowledge of the input distribution was assumed: the $16 \times 16$ subarray, the optimized subarray based on the actual error probabilities, and the subarray obtained by maximizing $\mathrm{SNR}_{1}$. Note that the simulation and $\mathrm{SNR}_{1}$ subarray curves are indistinguishable in both cases, indicating that estimation of the signal energies over the array does not result in any appreciable performance degradation. It also is evident that subarray optimization based on the simple $\mathrm{SNR}_{1}$ algorithm results in only slight losses, but succeeds in greatly reducing the complexity of the estimator; for $K_{b}=1.0$, the loss is less than $0.15 \mathrm{~dB}$ over the entire range considered in Fig. 11 .

\section{Summary and Conclusions}

The problem of improving the performance of ground-based optical receivers through the use of photoncounting detector arrays together with optimum signal-processing algorithms has been addressed. The optimum array detection algorithm was derived, and a simpler suboptimum structure based on the optimum 
algorithm also was defined. Exact and approximate expressions for the error-probability performances of these structures were derived, and simulations were carried out to verify the analytic performance calculations. Realistic sample functions of turbulence-degraded focal-plane signal distributions were generated using the Kolmogorov phase-screen algorithms described in [7], corresponding to moderate daytime turbulence (a coherence length of $4 \mathrm{~cm}$ ), and used to evaluate the performance of optimum and suboptimum array detection algorithms designed for PPM signals. Performance improvements of up to $5 \mathrm{~dB}$ were demonstrated over a single large detector designed to collect most of the turbulent signal, when operating in the presence of moderate to strong background radiation. The use of ideal adaptive optics in front of the detector, which concentrates most of the signal energy into a single detector element, was evaluated and found to provide approximately $3 \mathrm{~dB}$ of additional improvement at an error probability of 0.001 . It was also shown that, in cases of interest, the simpler suboptimum detector array algorithm performs nearly as well as the optimal array, with considerable savings in computational complexity.

\section{References}

[1] L. C. Andrews and R. L. Phillips, Laser Beam Propagation Through Random Media, Bellingham, Washington: SPIE Optical Engineering Press, 1998.

[2] R. M. Gagliardi and S. Karp, Optical Communications, New York: John Wiley \& Sons, 1976.

[3] D. L. Snyder, Random Point Processes, New York: John Wiley \& Sons, 1975.

[4] E. V. Hoversten, R .O. Harger, and S. J. Halme, "Communications Theory for the Turbulent Atmosphere," Proceedings of the IEEE, pp. 1626-1650, October 1970 .

[5] W. M. Hubbard, "Binary Detection in an Optical Twin Channel Receiver," IEEE Transactions on Communications Technology, vol. 19, pp. 221-223, April 1971.

[6] V. A. Vilnrotter, Optical Receivers Using Rough Reflectors, JPL Publication 85-25, Jet Propulsion Laboratory, Pasadena, California, May 1, 1985.

[7] P. Negrete-Regagnon, "Practical Aspects of Image Recovery by Means of the Bispectrum," Journal of the Optical Society of America, vol. 13, no. 7, pp. 15571576, July 1996.

[8] A. Papoulis, Probability, Random Variables and Stochastic Processes, New York: McGraw-Hill, 1965. 


\section{Appendix \\ Gaussian Approximation for Weighted Sum \\ of Poisson Random Variables}

Consider the characteristic function of the log-likelihood function defined in Eq. (13). Since the loglikelihood function is the sum of independent weighted Poisson random variables, each with characteristic function $[8]$

$$
\Phi_{m n}(\omega)=\exp \left\{\lambda_{m n} \tau\left[\exp \left(j \omega u_{m n}\right)-1\right]\right\}
$$

it follows that the characteristic function of the sum of independent weighted Poisson random variables is the product of the individual characteristic functions; hence, it is given by

$$
\Phi(\omega)=\exp \left\{\sum_{m=1}^{K} \sum_{n=1}^{L} \lambda_{m n} \tau\left[\exp \left(j \omega u_{m n}\right)-1\right]\right\}
$$

We can expand the exponential term (in the exponent) as

$$
\exp \left(j \omega u_{m n}\right)-1=1+j \omega u_{m n}-\frac{\omega^{2} u_{m n}^{2}}{2}+-\cdots-1=j \omega u_{m n}-\frac{\omega^{2} u_{m n}^{2}}{2}+-\cdots
$$

which is recognized as a power series in $(j \omega)$ with coefficients $\left(u_{m n}^{k} / k !\right)$. If we retain only the first two terms of the expansion, then Eq. (17) can be approximated as

$$
\begin{aligned}
\Phi(\omega) & \cong \exp \left\{\sum_{m=1}^{K} \sum_{n=1}^{L} \lambda_{m n} \tau\left[j \omega u_{m n}-\frac{\omega^{2} u_{m n}^{2}}{2}\right]\right\} \\
& =\exp \left\{j \omega \sum_{m=1}^{K} \sum_{n=1}^{L} u_{m n} \lambda_{m n} \tau-\frac{\omega^{2}}{2} \sum_{m=1}^{K} \sum_{n=1}^{L} u_{m n}^{2} \lambda_{m n} \tau\right\} \\
& \equiv \exp \left\{j \omega \eta-\frac{\omega^{2}}{2} \sigma^{2}\right\}
\end{aligned}
$$

This is recognized as the characteristic function of a Gaussian random variable with mean and variance $\eta$ and $\sigma^{2}$, respectively, where 


$$
\left.\begin{array}{rl}
\eta & =\sum_{m=1}^{K} \sum_{n=1}^{L} u_{m n} \lambda_{m n} \tau \\
\sigma^{2} & =\sum_{m=1}^{K} \sum_{n=1}^{L} u_{m n}^{2} \lambda_{m n} \tau
\end{array}\right\}
$$

Thus, the above approximation yields a continuous Gaussian random variable with mean and variance as defined in Eq. (A-5). 